$\xi=-1$

\title{
Probing Dynamic Mechanical Analysis and Atomic Force Microscopy of Polypropylene/Kaolin Nanocomposite
}

\author{
Dagaci Muhammad Zago ${ }^{1,3}$, Suzi Salwah Binti Jikan"*, Nur Azam Bin Badarulzaman², Nurun Najwa Binti Ruslan¹, \\ Awwal Hussain Nuhu1 ${ }^{4}$, Nazia Bano1 \\ ${ }^{I}$ Department of Physics and Chemistry, Faculty of Applied Sciences and Technology, Universiti Tun Hussein Onn Malaysia \\ ${ }^{2}$ Department of Materials and Design Engineering, Faculty of Mechanical and Manufacturing Engineering, \\ Universiti Tun Hussein Onn Malaysia \\ ${ }^{3}$ Department of Chemistry, Ibrahim Badamasi Babangida University, Lapai, Niger State, Nigeria \\ ${ }^{4}$ Department of Chemistry Bauchi State University, Gadau, Bauchi State, Nigeria \\ *Corresponding author E-mail: suzi@uthm.edu.my
}

\begin{abstract}
The Dynamic mechanical analysis (DMA) and Atomic force microscopy (AFM) studies were conducted and evaluated on polypropylene/kaolin (P/K) nanocomposite treated with maleic anhydride (MA) and dicumyl peroxide (DCP) as additives in an in-situ process. Two-roll mill was used in compounding of the nanocomposites while moulding were done by injection moulding machine. Investigation in to the effect of $\mathrm{K}$ and MA/DCP on the nanocomposites (NCs) indicates that interfacial interactions between PP and $\mathrm{K}$ as filler was eminent. DMA analysis reveals an increase in the storage modulus which was at maximum significantly in $\mathrm{P} / \mathrm{K} \mathrm{NC}$ with $3 \mathrm{wt} \%$ and decrease in damping factor tan $\delta$ also at $\mathrm{P} / \mathrm{K} \mathrm{NC}$ of $3 \mathrm{wt} \%$. The AFM study indicates that there was uniform and smooth surface roughness among the NCs. Thus, addition of MA/DCP on to P/K NC improves the reinforcing influence on the nanocomposites for better improvement.
\end{abstract}

Keywords: Atomic Force Microscopy; Dynamic Mechanical Analysis Polypropylene; Kaolin; Maleic Anhydride.

\section{Introduction}

In the preparation of polymer nanocomposites, nanoscale fillers are integrated into a pure polymer matrix to improve its properties if compared to that of the conventional polymer matrix. Subject to this characteristic transformation, two-dimensional platelet nanofillers are known for their interfacial interaction and dynamic mechanical improvement as well as dimensional stability [1]. Modified clay is extensively used as platelet nanofillers [2]. Kaolin used in this context is widely used in variable applications, such as filler in plastics, ceramics, paint extenders, paper coating and filling [3].

Research trends acknowledge the fact that degree of dispersion; either intercalation and/or exfoliation of organoclay into polymer matrices solidly impacts on the properties of the polymer/layered silicates nanocomposites [4]. In the recent time, scientific instruments used in characterization of polymer nanocomposites are either destructive in principle due to particles emission (radiation) like X-ray diffraction (XRD) or successive sample treatments like transmission electron microscopy (TEM) owing to sample preparation procedure involved. Alternatively, scarce research work has been conducted using a non-destructive techniques (AFM) which is similar to XRD [5]. To this effect significant discoveries yielded the fast development of PP production amid its thermoplastic equivalents inspired by its attractive combination of acceptable properties which allowed easy processing and diverse applications. These properties include outstanding chemical resistance, low cost and density, above all it accommodates heat distortion temperature (HDT) above $100{ }^{\circ} \mathrm{C}$. To this end, when PP is com- pounded with clay (kaolin) in the presence of additives (MA simultaneously with DCP) to prepare nanocomposites; improvement in HDT, dimensional stability, toughness and strength were achieved. PP, a multipurpose polymer being extensively involved in packaging, technical automotive parts, telecommunication cables and injection moulded products and compounds. The option to improve its properties can uncovered new prospects of application [6].

Dynamic Mechanical Analysis is basically employed to measure the transition in polymer layer nanocomposites that challenge detection using other types of techniques. The modification of materials are recorded via cyclic deformation. Three major parameters are core to DMA techniques. These include storage modulus (E'), which measures the elastic deformation of material. While the loss modulus $\left(\mathrm{E}^{\prime \prime}\right)$, is accountable for plastic response to deformation and the Tan delta damping factor $(\tan \delta)$ [7]. The ratio of the loss modulus to the storage modulus indicates transitions involving the motion in glass transition temperature of the nanocomposites. High value of $\mathrm{E}^{\prime}$, corresponds to improvement in compatibility and dispersion of nanocomposite as regards to the pristine PP $[8,9]$.

This work attempts to investigate the $\mathrm{P} / \mathrm{K}$ nanocomposites prepared using kaolin incorporated simultaneously with MA \& DCP. The P/K NCs obtained where then characterized by DMA and AFM for possible improved interfacial interaction and an upgraded material for superior application. 


\section{Experimental}

\subsection{Materials}

Kaolin in a white powder form was supplied by Kaolin (Malaysia) Sdn. Bhd. with chemical formula $\mathrm{Al}_{2}\left[\mathrm{Si}_{2} \mathrm{O}_{5}\right](\mathrm{OH})_{4}$ and chemical composition in wt $\%$ as $\mathrm{SiO}_{2}$ 57.633, $\mathrm{Al}_{2} \mathrm{O}_{3} 37.766, \mathrm{Fe}_{2} \mathrm{O}_{3} 0.86$, $\mathrm{MgO}$ 0.596, $\mathrm{CaO} 0.346, \mathrm{~K}_{2} \mathrm{O}$ 1.801, $\mathrm{TiO}_{2}$ 0.605, $\mathrm{P}_{2} \mathrm{O}_{5}$ 0.311. Polypropylene (PP) copolymer Titan pro SM-240 resin grade which is in pellet shape with density of $0.894 \mathrm{gcm}^{-3}$, melt flow index (MFI) $25 \mathrm{~g} / 10 \mathrm{~min}$ measured at $230{ }^{\circ} \mathrm{C} / 2.16 \mathrm{~kg}$ was used in this research and is supplied by Lotte Chemical Titan (M) Sdn. Bhd., manufacturer by Songhan Plastic Technology Co Ltd, Shanghai city, China. Both MA with molecular formula $\left(\mathrm{C}_{4} \mathrm{H}_{2} \mathrm{O}_{3}\right)$ and DCP, chemical formula $\left(\mathrm{C}_{18} \mathrm{H}_{22} \mathrm{O}_{2}\right)$ were obtained from Quality Reagent Chemical (QReC) Asia Sdn Bhd. Selangor Malaysia.

\subsection{Preparation of P/K Nanocomposites}

The $\mathrm{P} / \mathrm{K}$ nanocomposites were produced by In-situ process using range of different aggregates of kaolin $(1,3 \& 5$ wt.\%) as designated in Table I. by means of two roll mill model PW 3000 made in U.S.A. Independent addition of MA alongside DCP was made, also at similar ratio (1, 3 and 5 wt. \%). These gives us Polypropylene (PP), polypropylene/kaolin (PK), polypropylene/kaolin/maleic anhydride/dicumyl peroxide (PKMDCP) at $1 \mathrm{wt} . \%, 3 \mathrm{wt} \%$ and 5 wt. $\%$ as indicated in Table 1 . The processing temperature was kept at $190{ }^{\circ} \mathrm{C}$ throughout the compounding, with the processing rotor speed maintained at $50 \mathrm{rpm}$ for $10 \mathrm{~min}$. The nanocomposite produced was cooled down to ambient temperature. These were later crushed with the aid of plastic crusher machine into pellets of nanocomposites. The pellets were then moulded into dumb-bell shaped of $2 \mathrm{~mm}$ thickness by injection moulding machine model 109S050 obtained from Japan, at $190{ }^{\circ} \mathrm{C}$ and $20 \mathrm{MPa}$ per 10 minutes intervals, followed by cooling to room temperature at 5 MPa. The dumbbell samples prepared were utilized for the characterizations of the nanocomposites involving DMA measurement and AFM probing [10]. Dynamic mechanical analysis was performed using DMA Q800, of TA instruments, -waters LLC U.S.A. while the result analysed with the aid of TA instruments universal analysis 2000 software. The testing was conducted on a single cantilever bending mode at a vibration frequency of $1 \mathrm{~Hz}$ in a temperature ranging from $25-150{ }^{\circ} \mathrm{C}$ with heating rate at $5{ }^{\circ} \mathrm{C} / \mathrm{min}$ on a temperature ramp. The validation of surface roughness and topography of samples were done by AFM Bruker multimode instrument and NanoScope Analysis $1.50 \mathrm{r} 2$ at $1 \mathrm{~Hz}$ frequency.

\section{Results and Discussion}

\subsection{Dynamic Mechanical Analysis}

The DMA analyses performed on different NCs was to help study the PP \& P/K dynamic mechanical properties. It also provides evidence for the disposition behaviour of energy dissipation of the NCs [11]. This behaviour will uncover the effect of $\mathrm{K}$ and the compatibilizers on the heat distortion temperature as presented in Fig. 1(a), (b) and (c) which are storage modulus, (E'), loss modulus $\left(\mathrm{E}^{\prime \prime}\right)$, and the damping behaviour (internal friction) tan delta $(\tan \delta)$ respectively. The results of $E^{\prime}$ curves as a function of temperature for PP and all the NCs are given in Fig. 1(a). The values of $\mathrm{E}^{\prime}$ below $75^{\circ} \mathrm{C}$ are higher than that of pure $\mathrm{PP}$, but surprisingly the $\mathrm{E}^{\prime}$ of untreated $\mathrm{NC}$ falls lower at temperatures above $75^{\circ} \mathrm{C}$. This effect could be attributed to the absence or deficiency of compatibilizer which supports interfacial interaction between the PP matrix and the kaolin filler or possible agglomeration [12] Thus, from this report, it can be deduced that the NCs with compatibilizer have better compatibility and are stronger than the untreated $\mathrm{P} / \mathrm{K}$. Closer observation of the curve indicates that PKMDCP 3 reveals better compatibility and stiffness [13], since it possess higher E' than PKMDCP 1 and PKMDCP 5. Loss modulus is proportional to the energy dissipated during one loading cycle. It represents, for example, energy lost as heat, and is a measure of vibration.al energy that has been converted during vibration and that cannot be recovered [14]. Fig. 1(b) displays the $\mathrm{E}^{\prime \prime}$ as a function of temperature for PP and the NCs both treated and untreated. In the curve representing $E^{\prime \prime}$, the $E^{\prime \prime}$ of untreated $\mathrm{P} / \mathrm{K}$ has the least value among them, even lower than the pure PP. But in the case of treated NCs the one at 3 wt \% i.e. PKMDCP 3 have higher E" directly flow by that of PKMDCP 1 which is higher than that of PKMDCP 5, while PP has no sharp curve rather its cut across the entire curves [7]. Fig. 1(c) represents $\tan \delta$ which is the ratio of $E^{\prime} \& E^{\prime \prime}$ in a viscoelastic system and denotes the mechanic damping or internal friction as a measure of energy lost. High $\tan \delta$ value reflects material with high non-elastic strain components while low value of $\tan \delta$ signifies more elastic constituent. As revealed by the curves in Fig. 1(c) the NC PKMDCP 3 has lowest value of $\tan \delta$ indicative of higher elastic components with respect to other NCs. This is closely followed by PKMDCP 1 and PKMDCP 5 respectively, while the untreated P/K still misbehave cutting across the treated NCs from low $\tan \delta$ at lower temperature to high at high temperature. These characteristics present the untreated $\mathrm{P} / \mathrm{K}$ as lack of uniform interactions between PP and kaolin fillers [15]. However, pure PP has the highest $\tan \delta$ value as depicted by the curve with a wide difference through the temperature ranges. Thus it is evident that addition of kaolin filler with compatibilizer to PP have improved the elastic component of the $\mathrm{PP}$ which divulge the enhanced interfacial interaction.

\subsection{Atomic Force Microscopy}

AFM in its force modulation mode reveals a qualitative information about the local sample surfaces [16]. This test was use to probe the topography of all the NCs including the PP and $\mathrm{P} / \mathrm{K}$. The topography mapping results as shown in the images in Fig. 2. (a)-(c) for the captured surfaces for $\mathrm{P} / \mathrm{K}$ NCs give highlights between the differences in the topographic images which ranges from smoothness to roughness on the captured surfaces. The height and depth of the phase images as the basic morphology are also informed. The morphology as observed generally reveal different phases as indicated by uniformity of smoothness in Fig. 2(a) for PP. This smoothness is an indication of a low stiffness [16] due to the semi crystalline nature of pure PP i.e. presence of amorphous character. All the other $\mathrm{P} / \mathrm{K}$ NCs has different rate of roughness higher than that of PP. In all the P/K NCs as illustrated above in the 2D \& 3D images the PKMDCP 3 NCs with $3 \mathrm{wt} \%$ in Figure $2 \mathrm{c}$ has higher roughness which have showed that the assertion above as having better adhesion interactions.

Table 1: Formulation for Composite preparation

\begin{tabular}{|c|c|c|c|c|}
\hline $\begin{array}{c}\text { Composite } \\
\text { /Substrate }\end{array}$ & $\begin{array}{c}\text { Polypropylene } \\
\text { (PP) wt.\% }\end{array}$ & $\begin{array}{c}\text { Kaolin } \\
(\mathrm{K}) \\
\text { wt.\% }\end{array}$ & $\begin{array}{c}\text { Maleic } \\
\text { anhydride } \\
\text { (MA) wt.\% }\end{array}$ & $\begin{array}{c}\text { Dicumyl } \\
\text { peroxide } \\
\text { (DPC) } \\
\text { wt.\% }\end{array}$ \\
\hline PP & 100 & - & - & - \\
\hline PK & 97 & 3 & - & - \\
\hline PKMDCP 1 & 95 & 3 & 1 & 1 \\
\hline PKMDCP 3 & 91 & 3 & 3 & 3 \\
\hline PKMDCP 5 & 87 & 3 & 5 & 5 \\
\hline
\end{tabular}



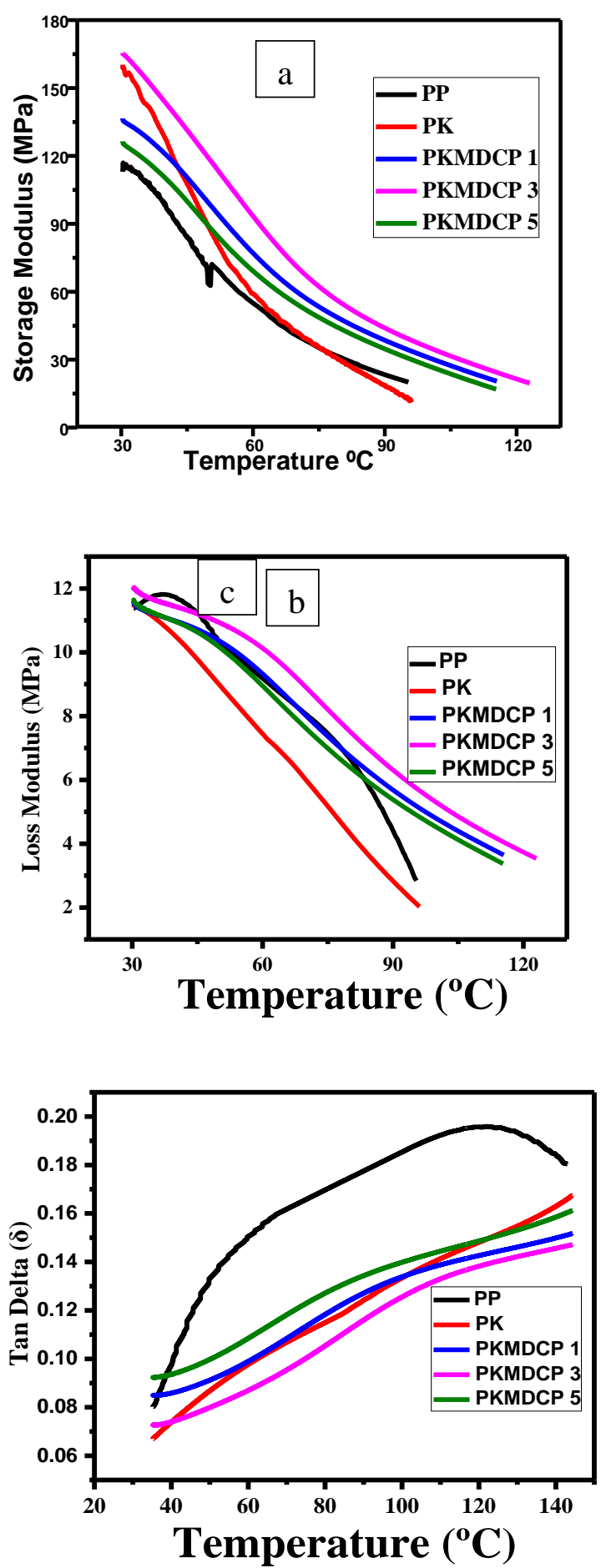

Fig. 1: DMA spectra of PP, treated and untreated nanocomposites (a) storage modulus ( $\left.E^{\prime}\right)(b)$ loss modulus $\left(E^{\prime \prime}\right)(c) \tan \delta$

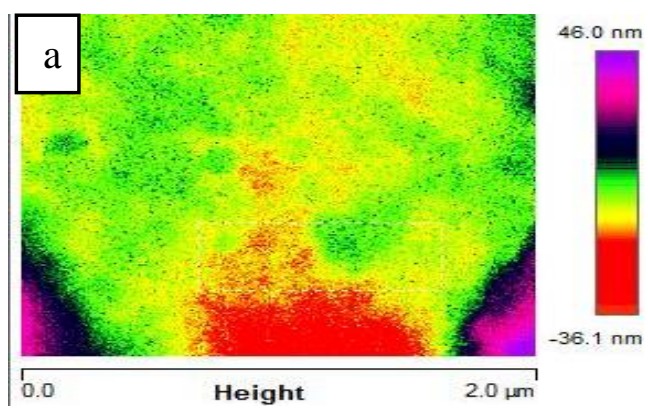

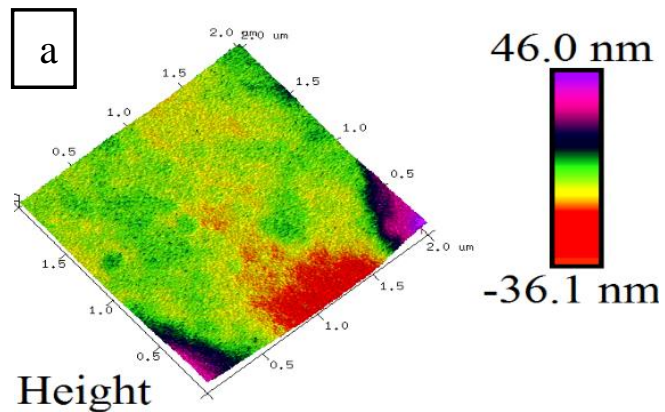
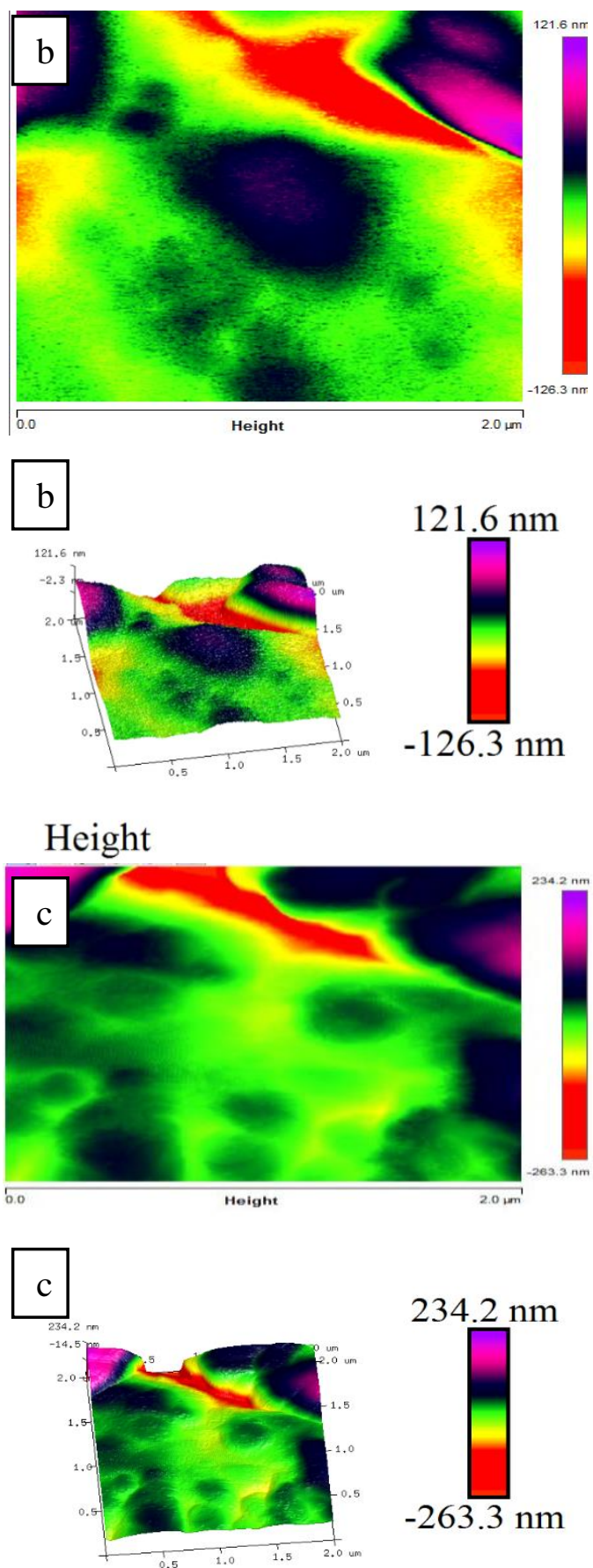

$234.2 \mathrm{~nm}$

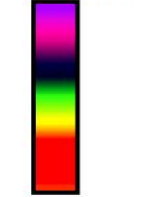

$-263.3 \mathrm{~nm}$

Height

Fig. 2: AFM Topography images of (a) PP (b) PK (c) PKMDCP 3 


\section{Conclusion}

This investigation accentuated the use of filler (kaolin) and compatibilizer (MA \& DCP) as additives in the reinforcement of the nanocomposites which boosts the dynamic mechanical properties of the NC as indicated in all treated NCs (PKMDCP 1, PKMDCP 3, \& PKMDCP 5) but at its best in PKMDCP 3. The storage modulus increases with addition of additives and decrease in $\tan \delta$ both of which improves the elastic behaviour of the P/K NCs. These findings indicate benefit for future development and applications.

\section{References}

[1] Aoyama S, Park YT, Macosko CW, Ougizawa T \& Haugstad G AFM (2014). Probing of Polymer/Nano fi ller Interfacial Adhesion and Its Correlation with Bulk Mechanical Properties in a Poly(ethylene terephthalate) Nanocomposite. Pubs.acs.org/Langmuir AFM 30, pp. 12950-12959.

[2] Durmus A, Sariboga V \& Faruk MAO (2013), Investigation of Thermal , Rheological , and Physical Properties of Amorphous Poly ( ethylene terephthalate ). Organoclay Nanocomposite Films, pp. 2490-2501.

[3] Ahmed NM \& El-sabbagh SH (2015), The Influence of DopedKaolin on the Properties of Styrene Butadiene Rubber Composites, 3, pp. 1-19.

[4] Liu TX et al. (2003), Morphology, thermal and mechanica behavior of polyamide 6/layered-silicate nanocomposites. Compos. Sci. Technol. 63, pp 331-337.

[5] Morales AR., Paiva LB, De, Zattarelli D \& Guimarães TR (2012), Morphology, Structure and Mechanical Properties of Polypropylene Modified with Organophilic Montmorillonite, 22, pp. 54-60.

[6] Zaman HU, Hun, PD, Khan RA \& Yoon KB (2014), Polypropylene/clay nanocomposites, J. Thermoplast. Compos. Mater. 27, pp. 338-349.

[7] Benmesli S \& Riahi F (2014), Dynamic mechanical and therma properties of a chemically modified polypropylene / natural rubber thermoplastic elastomer blend. Polym. Test. 36, pp. 54-61.

[8] Scobbo JJ (1991), Dynamic Mechanical Analysis of

[9] Ambrogi V, Carfagna C, Cerruti P \& Marturano V (2016), Additives in Polymers. Modification of Polymer Properties, pp. 140.

[10] Dagaci ZM, Jikan SS, Nur Azam B, Shehu, Y \& Awwal HN (2017), Effect of Additives on Polypropylene Kaolin Composite Prepared via In-situ Process Effect of Additives on Polypropylene / Kaolin Composite Prepared via In-situ Process. J. Sci. Technol. 9, pp. 1923.

[11] Komalan C, George KE, Kumar PAS, Varughese KT \& Thomas S (2007), Dynamic mechanical analysis of binary and ternary polymer blends based on nylon copolymer / EPDM rubber and EPM grafted maleic anhydride compatibilizer. 1, pp. 641-653.

[12] Eguiazabal J, Granado A \& Naz J (2010), Compatibilized blends of polypropylene and a poly ( amino ether ). Soc. Plast. Eng., pp 1-3.

[13] Macagnano A, New Applications of Nanoheterogeneous Systems. Nanostructured Polymer Blends Elsevier Inc. (2013), pp. 450-493.

[14] Ehrenstein GW, Riedel G \& Trawiel P (2004), Dynamic Mechanical Analysis. Therm. Anal. Plast. Theory Pract, pp. 236299.

[15] Venegas C (2010), Polypropylene/clay nanocomposites Synthesis and characterization. J. Chil. Chem. Soc. 4, pp. 2-6.

[16] Gao SL \& Mäder E (2002), Characterisation of interphase nanoscale property variations in glass fibre reinforced polypropylene and epoxy resin composites, Compos. - Part A Appl. Sci. Manuf. 33, pp. 559-576. 